partic du courant étant transforméc en courants diphasés 42 périodes pour l'éclairage.

En aval des ouvrages, le rógime du fleuve serait maintenu tel qu'il est sans l'existence du barrage. Le régime serait réglé de manière à ne pas troubler le fonctionnement des usines existantes el à ne pas aggraver les variations journaJières de débit provoquées par les usines d'amont.

Le grandiose projet du " Rhône à Paris " apporterait à la capitale un appoint considérable de puissance et lui permettrait d'economiser quelques centaines de milliers de tonnes de houille, achetécs actucllement à l'étranger. Ce scrait, comme l'a dit M. Loucheur, un nouveau bassin houiller áans Ie centre de la France, dont la valcur représente, en admettant que $x$ kilogr. 2 de houille produit un kilowatt-heure, près de 1.600 .000 tonnes de houille par an.

(A suivre).

H.-L. Bermaridin, Ingénieur des Arts el Manufactures.

\section{LES INDUSTRIES DE L'AZOTE}

La Chambre Syndicale des Forees Hydranliques a organisé, il y a deux ans, à l'instigation et sous la direction de l'un des plus actifs de ses administrateurs, M. Georges Coutagne, un nouveau service technique : la publication mensuelle des numéros, dates de demande, noms des demandeurs ot titres do tous les brevets français concernant l'électrochimic, l'électrométallurgie ot les industries qui s'y rattachent. Une analyse sommaire est jointe, en oulre, pour ceux de ces brevets qui concernent plus spécialement la chimie industrielle, c'est-à-dire la mélallurgie, l'électrométallurgic, l'dectrochimie et la grande industric chimique.

La dircction de La Houille Blanche a lenu à faire bénéficier ses abonnés de celte publication, et par suite d'arrangements intervenus avec la Chambre Syndicale, el d'une participation aux frais de ce service, notre Revue a élé autorisée depuis un an à reproduire in extenso les listes do brevets et analyses publićes mensuellement dans le Bulletin de la Chambre syndicale des forces hydrauliques.

Nous désirons signaler aujourd'hui le grand intérêt que présentera bientót le travail de synthèse qui doil faire suite au travail d'analyse long, minuticux, et assurément assez ingrat, qui a éé entrepris depuis deux ans par lo nouveau Service des brevets de la Chambre syndicale. Si l'on se borne en effet, à considérer la liste des brevels publiés par cxemple pendant un mois, l'intérêt que présente une telle liste est cêrlainement très spécial. Mais il n'en est plus de mểnó si l'on considère la liste, avec classificalion mélhodique, te tous les brevels publiés pendant une annéc, ou pendant deux années conséculives. Or, éest pnócisément une telle liste, pour les deux années rgr et rgra, qui vient de paraître dans le dernier Bulletin de ta Chambre syndicale.

Nous donnons ci-après un extrait de cette table méthodique, dans laquelle les brevets sont classés à un point de vue strictement industriel, mais, pour faciliter les recherches, dans l'ordre alphabétique des éléments chimiques. Cet extrait, très restreint, concerne seulement l'azote. Les brevets relatifs à la préparation et à la fixation de cet élément, ou encore à la transformation de combinaisons azotécs en d'autres combinaisons azotées, sont classés en qualorze paragraphes. Mais plusieurs de ces paragraphes seront subdivisés à leur tour, dans les tables méthodiques beaucoup plus ćtendues, c'est-ì-dire comprenant un plus grand nombre d'an- nées; que sc propose d'établir et de publier ultérieurèment M. Georges Coutagne.

Nous faisons des voux pour que la. Chambre syndicale des forces hydrauliques donne à son nouveau service des brevels une extension croissante, et pour que nous puissions avoir bientôt, grâce ì clle, une table générale mélhodique de tous les brevets français relatifs a l'industrie chimique, classés par industries el même par opérations industriclles distinctes de chaque industrie, remontant jusqu'au $x^{\text {er }}$ janvicr $x$ gos, et mise à jour tous les ans ou tous Jes deux ans. Une telle publication, constituerait un instrument d'etudes pour le passé, of de recherches pour l'avenir, excessivement précicux, et susceptible de faroriser beaucoup to développement de l'industrie chimique en France.

\section{EXTRAIT DE LA TABLE DES BREVETS}

1. Azote non combiní. - Extraction de l'azote de l'air: fabrication des mélanges d'azote et d'hydrogène.

423224.9 février 1910. Société "L'air liquide". 423462. ar novembre 1910. The Nitrogen Company, 497 834. 28 mars 1911. Sociéle générale des nitrures. 430 092. 24 mai ygu. Georg Kaszner. 430 og3. 24 mai rgn. Georg Kaszuer. 433 339. lí aoù rom. The Nitrogen Company. 4.10 702. 9 mai 19 r. George-Frangois Jaubert. 444 044 . 20 mai 1912. Dieffenbach el Moldenhauer. 446560.26 juillet $9_{912}$. Industriegas Gesellschaft, ek.

2. Azote (Fixation). - Synthèse des oxydes d'azote A teMpératures peU Élevées.

437397.9 décembre I91 I. Nö̈l Lecesne. 443620 . 9 mai y 912 . Russ el Ehrlich.

\section{Azote (Fixation), - Synthese des oxydes dizote} A HAUte température, NoN blectrique.

f20 Ir2 7 septembre 19ro. Fricdrich Hausser.

43 × 93.6 juin $x$ gr. Alfred Cobbell.

4328 ro. 25 juillel rgr. Société : Nitrogen limited.

435397 . 18 oclobre 19ı. Kanl Kaiser.

440218.27 avril $\mathrm{x} 9$ 1r. Cio Bordelaise de produits chimiques. $446269 . x_{9}$ juillet $191 \%$. Irónć Dupont.

4. Azote (Fixation). - Synthèse des oxydes d'azote A HAUTE TEMPÉRATURE, ELECTRQUE.

357 358. 3o août igo5. Badische Anilin \& Soda Fabrik.

$5559 . \quad 9$ décembre 1 go5. Badische Anilin \& Soda Fabrik.

$62 \times 9.26$ avril 5906 . Badische Anilin \& Soda Fabrik.

649 r. 4 juillel xgo6. Badische Anilin \& Soda Fabrik. I3 750. 26 janvier 191x. Badische Anilin \& Soda Fabrik. 420 25\%. 3 septembro 1010. Salpetersaüre Ind. Gesclischaft. 120524. 77 septembre 19ro. Sociélé : Le Nitrogène.

423755.7 décembre 1910 . Eugène Wassmer.

425725 . 8 février r 9 rx. Haryy Pauling.

426306 . 2x février IgIr. Elektrochemische Werke.

427745 . 25 mars 191. Carl Fabian Richert yon Koch.

4299 ro. 18 mai rgIr. Elektrochemische Werke.

433 г12. 9 août 19 rr. Société : Nobel et $\mathrm{C}^{\text {ie }}$.

435733.23 octobre 19ז. Carl Fabian Richert voü Koch.

438783.8 janvier 1912. Société : Le Nitrogène.

443 729. ir mai igr2. Badische Anilin \& Soda Fabrik.

445489.7 juin r912. Salpetersaüre Industric Gesellschaft: 446 229: is jutllet. Andriessens et Scheidemandel.

5. Azote. - Transfonmation des composés oxygénés 
de l'azote en d'autres composés oxygénés : traitement

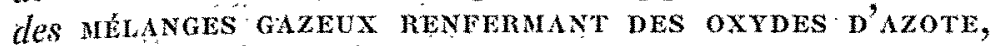
et traitement des acides, nitriles ou nitrates en dérivant. 412788. x janvier rgro. Badische Anilin \& Soda Fabrik.

I3 39 4. 9 novembre rgro. Badische Anilin \& Soda Fabrik. 420803.26 septembre rgro. Harry Pauling.

420 804. 26 soplembre r9ro. Harry Pauling.

$421022, I^{\text {er }}$ octobre 19ro. Sociélé : Le Nitrogène.

421 313. 18 décembre 19og. Société : Ie Nitrogène.

I35ig. I ${ }^{\text {cr }}$ octobre r9ro. Société : Le Nitrógène.

42275 . 8 novembre xpro. Carl Christian Scharb.

123760.8 décembre r 9 ro. Farben fabrik Bayer $\& C^{\circ}$.

424598 . / janvicr $\operatorname{rgr}$. Société : Le Nitrogènć.

4259 r3. 18 avril igro. Taraud et Truchot.

I 4 299. 20 août rgro. Taraud et Truchot.

425 997. 13 févier rgrx. Norsk Hydro-Eloktr. Kv.

428657 . 18 avril rgIr. Meister Lucius \& Bruning.

$430 \mathrm{I3}$. 2/ mai rgrx. Salpetersaüre Industrie Gesellschaft.

$430 x+3$. 34 mai r $9^{1}$. Salpetersaüre Industrie Gesellschaft.

43 1006 . I3 juin r9 . Elektrochemische Werke:

13 r $125.1^{\text {or }}$ septembre I9ro. Raoul Delplace.

$43 \times 427$ ro septembre rgro. Taraud et Trachot.

$432252 \times 3$ juillet rgr. Elektrochomische Werke.

43\% 990. 4 août Igrr. Meister Lucius \& Bruning.

433 4 10. Ig août 19 r. Meister Lucius \& Bruning.

433502 2 r août r91 . Meister Lucius \& Bruning.

43549\%. 17 octobre rgrr. Hale et Scott:

4h1697. 30 mai rgr r. Théophile Schloesing.

4/1 $\mathrm{r}^{2}$ 06. 25 mars igr2. Elektrochemische Werke.

442514 . ar juin rgrx. Société norvégienne de l'azote.

4h5 826. 6 juillet rgra. Norsk Hydro-Elektr. Ky.

6. Azote. - Fixitron sur le Silicrum, Azoture et carboazoture de silicium.

43r 182 . r6 mai rgrr. Sinding-Larsen et Storm.

433 g95. ia novembre igro. Otokar Serpek.

438 209. 8 mans r 9 rr. Societé générale des nitrures.

15 344. ro avril r 9 rix. Société générale des nitrures.

438 248. 9 mars r gr r. Société générale des nitrures.

7. Azote. - Fixatron sur L'Aluminium. Nitrure d'aluminium.

426868. r3 mai rgro. Otokar Serpek.

427 066. 18 mai igro. Otokar Serpek.

I3 958. $x^{\text {er }}$ juin r 9 ro. Otokar Serpek.

14 338. 3r aout rgro. Otokar Serpek.

427 rog. ig mai rgro. Otokar Serpek.

427 110. 19 mai igro. Otokar Serpek.

430553. İ a aût r 9 ró. Otokar Serpek.

14589. 28 octobre rgro. Otokar Sérpek.

if 830.8 décembre rgro. Otokar Serpek.

i5 $8_{2} 3$. 4 août r 9 Ir. Société générale des nitrurce

430. 832.23" août 1910. Otokar Serpek.

ił36 596 . 26 janvier r $9 \mathrm{rr}$. Société générale des nitrures.

$436789 .{ }^{8 *}$ février rgr. Georges Coutagne.

I5 15. 25 février r 9 1r. Georges Coutagne.

I5 619. 27 mai rgrr. Georges Coutagne.

437 504. 20 févricr r 9 r. Gcorges Coutagne.

15 774. 29 juin I9r. Georges Coutagne.

438 200. 8 mars rgrr. Société générale des nitrures.

$1534 \%$. Io avril 19 r. $x$. Société générale des nitrures.

438 248. 9 mars igr. Société générale des nitrures.

438 098. 28 mars rgr . Société générale des nitrures.

444316.4 août igri. Société générale des nitrures.

$4: 6978$ : 7 août t 9 r2: Leflaive ei Compagnie.
8. Azóte. - Fixition sur le carbure de chlchum. Industrie de la Cranamoe : fabrication, purification, granulation, etc. (Non compris les transformations chimiques de la cyanamide en a'autres produits azotés tels que l'ammoniaque).

423072.4 février 19 ro. Louis Devaucelle.

423562 . ro décembre 1910. Théodor Knösel.

430 06o. 23 mai rgur. Karl Schick et la Sociélé, otc.

tho 388. $x^{\text {er }}$ juin igrr. Rudolphs el Gustaf Thisell.

43/4 953. 6 octobre r 9 r. Gustavo Scialoja.

412 oo4. $x^{\text {er }}$ avril 1912. Ferben fabriken F. Bayer \& $\mathrm{C}^{\circ}$.

4h4 933 . 13 juin $19 \times 2$. Albert Renault.

44542.28 juin rgr2. Oscar Fredrik Carlson.

9. Azote. - Fixation sur des filments autres que $\mathrm{SI}, \mathrm{AL}_{\mathrm{L}}$, $\mathrm{er} \mathrm{C}_{\mathrm{A}}$ : fabrication des cyanures alcalins à partir de l'azote gazeux, etc.

422963 . 4 novembre 1910 . Swan et Kendall.

425025.6 octobre 99 ro. The Nitrogen Company.

495699. it/ octobre r9ro. The Nitrogen Company.

438 248. 9 mars r 9 rr. Sociélé générale des nitrures.

439244 . 22 janvier rgr2. Franck et Fincke.

$43987^{2}$. 8 février rg12. Schweitzer of Fritz Irauff.

10. Azote. (Fixation). - Syntuise de hamaningue à partir de l'azote el de la vapeun D'EAU, avec un azoture fixe comme intermédiaire.

429 r fo. 29 avril $x_{9} \times 1$. Louis Petit-Devaucelle.

436 7ro. 3o janvier igrr. Georges Coulagne.

437 168. ro févricr rgrr. Sociétó générale des nitrures.

4/55 506. 27 juin 19г2. Paul Weber.

11. Azote (Fixation). - Synthése de lammoniaque a PARTIR DE SES ELLEMENTS.

423755.7 décembre I91o. Wassmer.

425.099 .24 nov. I9ro. Badische Anilin und Soda Fabrik.

I5 984. 24 mai rgra. Badische Amilin und Soda Fabrik.

4.25 952. I9 avril igro. Brochet et Boiteau.

429696 . r3 mai rgrr. Badische Anilin und Soda Fabrik. 430595 . 13 aoùt 19tr. Arthème Matignon.

13r 295. rg juin rgrr. Badische Anilin und Soda Fabrik. 4,36 472. 16 nov. 9 $9 \times x$. Badische Anilin und Soda Fabrik. 436 680. 23 nov. rgr. Badische Anilin und Soda Fabrik. 43926 r. 22 janv. r912. Badische Anilin und Soda Fabrik. 440217.27 avril $19 \mathrm{r}$. $\mathrm{C}^{\mathrm{io}}$ bondelaise de produits chimiques. 15525. r2 mai rgrx. Cordelaise de produits chimiques. 44 I 695. 23 mars Igr.2. Badische Anilin und Soda Fabrik. 36259.26 juillet 19 i2. Badische Anilin und Soda Fabrik. 444 \{55. 3o mai rgra. Badische Anilin und Soda Fabrik.

12. Azote (Fixition). - Syntmìse du cyanogine et DE L'ACIDE CYANHYDRIQUE.

435308 . 2o décembre 1910. Société générale des nitrures. 436 I85: 16 janvier rgrr. Guignard el Watrigant.

. 5080 , ro février xgrr. Guignard et Watrigant.

436 20r. 2 août rgr r. Chem. Fabr. Griesheim Elektron. 1336828 . 25 novembre 5911 . Alois Helfenstein.

13. Ażote. - Transponmations de produits azoles en

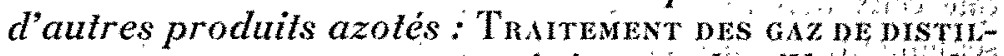
Latron des combustubes minéraux, houille, tourbe, etc., ou des matières ayant servi à l'épuration de ces gaz, en vue de la récupération des composés amoNiacaux. V. à Soufre, pour le traitément de ces mêmes gaz ou matières en vue de la récupération du soufre. 
420 8o6. 26 septembre roro. Karl Burkheiser.

426 r25. I6 février rgr . Mason (The suJphate... elc.).

427 o65. I8 mai I9ro. Soc. industr. de produits chimiques.

14 046 . 24 juin rgro. Soc. industr. de produits chimiques.

15307.29 mars 9 r. Soc. industr. de produits chimiques.

429 708. п3 mai r91. Karl Burkheiser.

43 г $2 / 4$. I7 juin rgr. Walther Feld.

13259o. 17 juin rg⿵. Walther Feld.

434 232. 5 septembre y gro. Braunschild el Chapiro.

1/4662. is novembre igro. Braunschild ef Chapiro.

434 673. 2 décembre rgro. Gouthière et $\mathrm{C}^{\mathrm{ie}}$, et Dueancel.

55 449. 2 mai rgr. Gouthière ot $\mathrm{C}^{\mathrm{ie}}$, et Ducancel.

436768.28 octobre r91. Gewerkschaft des Steink. Jothr.

$437257 . \quad 7$ décembre igx. Fabry.

$43_{9} 365$. 4 avril rgr. Gouthière ol $\mathrm{C}^{\mathrm{ie}}$, et Ducancel.

4 $4033 \mathrm{r}$. er $^{\text {mai }}$ I $9 \mathrm{~s}$. Burgevin.

44 5 53.20 mars igr2. Karl Burkheiser.

445 т62. I9 juin r9 2. Frederic William Burstall.

445556.28 juin r9 9 . Karl Burkhoiser.

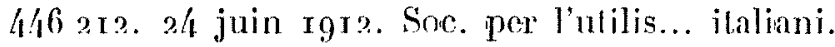

14. Azote. - Transformations de produits azolés en d'autres produits azotés (non classés déjà aux séries $5^{\mathrm{e}}$ et $13^{\circ}$ de l'azote).

41/682. 9 avril r9ro. Freth ol Edwin Cocksedge.

r35 го. 5 décembre rgıo. Freeh el Edwin Cockselge.

4 í9 $78 \% 27$ août 1910. Karl Kaiser.

420258.7 septembre s 910 . Erust. IIenss.

42 85 г. 26 octobre г9 ro. Edgar Arthur Asheroft.

426307.2 f fevier rga r. Flektrochemische Werke.

428 or 4 . I juin rgro. Braunschild el Chapiro:

429 708. I8 mai r9ı. Karl Burkheiser.

430 93r. r3 juin rgra. Freeth et Cocksedge.

43r 058. I4 juin igr. Freeth et Cocksedge

43r o88. 3 r août r 9ro. Gouthière et Ci, el Ducancel.

433 3o6. 3 août rgr I. Alby. U. C. F. Ld.

135397 . 8 octobre r9 1... Karl Kaiscr.

435476 . 28 seplembre ig r. The New Jersey Zine C.

436 185. 16.janvier ig1 Guignard el Watrigant.

I5 o80. 1o fevier rgir. Guignand el Watrigant.

436768.28 oclobre 191 . Gerverkschaft des St. Iolhr.

437 I68. to février ig 1 r. Sociélé générale des nilrures.

438 э48. 9 mars rgr. Société générade des nilrures.

438487. 2 décembre 1911. Chemische Fabrik ron Jeyten

$4389_{965}$. т3 janvier ig12. ITenrich sulzer.

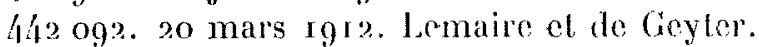

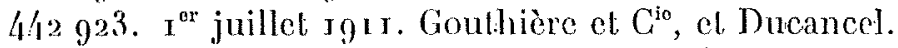

r6 297. $\mathrm{r} 7$ octobre rger. Goulhiere et $\mathrm{C}^{\mathrm{io}}$, ol Durancel.

4,5 857. r5 septembre rg1 . A. de Montlaur.

4h7 ro6. ro août rger. Emil Collet.

4473 : r. 7 août igr2. Edgar Arthur Asheroft.

Cette nomenclature donne une idée frappante de l'incomparable activité que les chercheurs déploient pour atteindro la solution de ce problème si important posé dès les origrines de la sciene: chimique : l'industrialisalion de l'azote. Fn remarquant que celle longue liste de brevets ne comprend que ceux délivrés ces deux dernières annécs, el en France seulement, tandis qu'à l'étranger, notamment en Allemagne, dans les Pays Scandinaves, en Angleterre et aux Etats-Unis, benucoup d'autres brevets ont été pris qui n'ont pas ćté de. mandés en France, on juge quelle doit être la somme considérable des Iravaux s'accumulant.
La semence jetée par celle foule de travailleurs contient assurément de l'ivraie, c'est-à-dirc un certain nombre de conceplions pratiquement irréalisables, mais aussi des idées justes et des découvertes de valeur - les bons grains - et il ne peut y avoir de doute : la moisson sera belle. Nous avons la conviction que te champ de la houille blanche fera pour sa parl une abondante récolte.

E.-F. Côte.

\section{TRAMWAYS ÉLECTRIQUES}

\section{Voitures à plancher surbaissé et sans marchepied des " New-York-Railways"}

Une des Compagnies qui desservent New-York, la NewYork Railways Company, a, cetle année, mis en service une voiture sans marchepied qui, ainsi qu'on s'cn convaincra facilement par l'aspect de la figure $I$, constitue l'innovation la plus originale el la plus hardie qui ait été depuis long-

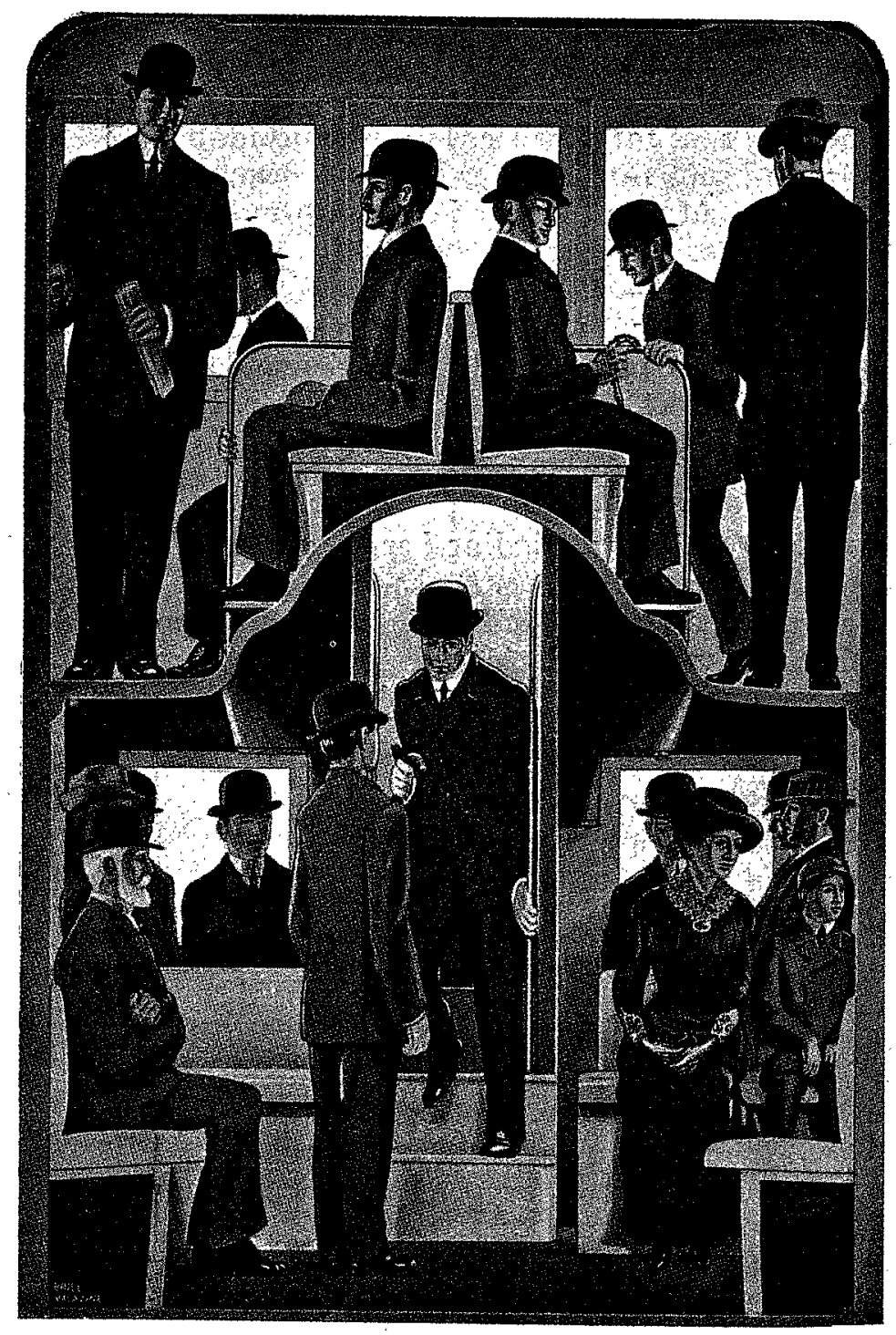

FIG. I. - COUPE TRANSVERSALE DE LA VOITURE.

temps réalisée dans la construction des voitures. On sait ì quels accidents de toute sorte donnent lieu les marchepieds; te plus souvent peu ćludićs, des voitures de lramways, et quelle gêne et quels relards ils occasioment, trop souvent it la monlée, ou à la descenle. Les ingénieurs de New-York onl appliqué au mal le remède le plus radical qui soit a ils ont purement et simplement supprimé le marchepied, of 